\title{
Impact of a Single 36 Hours Prolonged Fasting Period in Adults With Type 1 Diabetes - A Cross-Over Controlled Trial
}

\section{OPEN ACCESS}

Edited by:

Annette Schürmann German Institute of Human Nutrition Potsdam-Rehbruecke (DIfE), Germany

Reviewed by: Dubravka Jurišić Eržen, University of Rijeka, Croatia Bettina Berger. Witten/Herdecke University, Germany

*Correspondence: Othmar Moser othmar.moser@uni-bayreuth.de Harald Souri ha.sourij@medunigraz.at

Specialty section: This article was submitted to Clinical Diabetes, a section of the journal Frontiers in Endocrinology

Received: 20 January 2021 Accepted: 21 June 2021 Published: 06 July 2021

Citation:

Moser O, Eckstein ML Mueller A, Tripolt NJ, Yildirim $H$ Abbas F, Pferschy PN, Goswami N, Aberer F, Obermayer A, Pieber TR,

Kojzar H, Sourij C, Brunner M, Niedrist T, Herrmann $M$ and Sourij $H$ (2021) Impact of a Single 36 Hours

Prolonged Fasting Period in Adults With Type 1 Diabetes - A

Cross-Over Controlled Trial.

Front. Endocrinol. 12:656346. doi: 10.3389/fendo.2021.656346

\begin{abstract}
Othmar Moser ${ }^{1,2 *}$, Max L. Eckstein ${ }^{1,2}$, Alexander Mueller ${ }^{1,3,4}$, Norbert J. Tripolt ${ }^{1}$, Hakan Yildirim ${ }^{1}$, Farah Abbas ${ }^{1}$, Peter N. Pferschy ${ }^{1}$, Nandu Goswami ${ }^{5}$, Felix Aberer ${ }^{1}$, Anna Obermayer ${ }^{1}$, Thomas R. Pieber ${ }^{1}$, Harald Kojzar ${ }^{1}$, Caren Sourij ${ }^{6}$, Martina Brunner ${ }^{1}$, Tobias Niedrist ${ }^{4,7}$, Markus Herrmann ${ }^{4,7}$ and Harald Sourij ${ }^{1 *}$

${ }^{1}$ Interdisciplinary Metabolic Medicine Trials Unit, Division of Endocrinology and Diabetology, Department of Internal Medicine, Medical University of Graz, Graz, Austria, ${ }^{2}$ Division of Exercise Physiology and Metabolism, Department of Sport Science, University of Bayreuth, Bayreuth, Germany, ${ }^{3}$ Exercise Physiology, Training \& Training Therapy Research Group, Institute of Sports Science, University of Graz, Graz, Austria, ${ }^{4}$ Austria Clinical Institute of Medical and Chemical Laboratory Diagnostics, Medical University of Graz, Graz, Austria, ${ }^{5}$ Division of Physiology, Otto Loewi Research Center, Medical University of Graz, Graz, Austria, ${ }^{6}$ Division of Cardiology, Medical University of Graz, Graz, Austria, ${ }^{7}$ Clinical Institute of Medical and Chemical Laboratory Diagnostics, Medical University of Graz, Graz, Austria
\end{abstract}

Prolonged fasting has shown beneficial effects in healthy individuals and in people with chronic diseases. In type 1 diabetes, the effect or even the feasibility of fasting is unclear. We aimed to assess the impact and safety of prolonged fasting in adults with type 1 diabetes. Glycemia was assessed during overnight fasting (12 hours) vs. prolonged fasting (36 hours) via an intermittently-scanned continuous glucose monitoring system. Anthropometric data, metabolic and hormonal markers were compared between both trial arms. After each fasting period, a $75 \mathrm{~g}$ oral glucose tolerance test was performed and plasma glucose levels and hormones were assessed. Data were compared via paired ttests and mixed-model regressions ( $p \leq 0.05)$. Twenty individuals with type 1 diabetes (7 females) with a mean $\pm \mathrm{SD}$ age of $35 \pm 11$ years, body mass index (BMI) $24.8 \pm 2.8 \mathrm{~kg} / \mathrm{m}^{2}$ and $\mathrm{HbA}_{1 \mathrm{c}} 54 \pm 7 \mathrm{mmol} / \mathrm{mol}$ were included. Hypoglycemia/hour (70 mg/dL; $\left.<3.9 \mathrm{mmol} / \mathrm{L}\right)$ was similar in both trial arms (12 hrs: $0.07 \pm 0.06$ vs. 36 hrs: $0.05 \pm 0.03, p=0.21$ ). Glycemic excursions during the oral glucose tolerance test were not different after the two fasting periods. Beta-hydroxybutyrate levels were higher after prolonged fasting $(p=0.0006)$. Our study showed that people with type 1 diabetes can safely perform a 36 hours fasting period with a low risk of hypoglycemia and ketoacidosis.

Clinical Trial Registration: DRKS.de, identifier DRKS00016148. 


\section{INTRODUCTION}

Reducing food availability via caloric restriction (fasting) was shown to improve health, improve markers of aging and ameliorate clinical outcomes in several diseases (1-3). In general, caloric restriction can be categorized into intermittent fasting and time restricted feeding (4). Intermittent fasting is defined by repeated periods with low or no energy intake that might be further classified by alternate day fasting, alternate day modified fasting and fasting or modified fasting on two days per week. Periodic fasting is characterized by fasting for two or more days. Time restricted feeding can be defined by eating patterns, which are restricted to a short ( $<8-10$ hours) interval each day (4). Different metabolic, hormonal and cellular effects have been described to be associated with fasting (5): energy sources switch to the utilization of fatty acids and ketone bodies (6), improvements in insulin sensitivity $(7,8)$ and cells activate pathways improving intrinsic defenses against oxidative and metabolic stressors (9). Furthermore, it was shown that intermittent fasting is an effective life-style intervention to lower bodyweight and improve waist circumference in overweight people with type 2 diabetes (7). Since overweight and obesity in children (10) and adults with type 1 diabetes increases and equal those of the general non-diabetes population (11), the methodology of intermittent fasting might also be applied in type 1 diabetes. In people with type 1 diabetes, fasting might lower the need for exogenous insulin (12), stabilize glucose levels in a near normoglycaemic range, lower bodyweight and body mass index (BMI) and lower the total carbohydrates intake (13).

Taking these positive effects of fasting and its potential to improve cardio-vascular outcomes into account, people with type 1 diabetes do follow dietary regimens more often. Those include also strategies incorporating longer fasting periods, however, data on the impact of fasting interventions on safety, namely hypoglycaemia and diabetic ketoacidosis and the change in the insulin dose needed following a prolonged fasting period in people with type 1 diabetes are scarce. Especially, physiological ketosis needs to be separated from diabetic ketoacidosis (13): a regular blood ketone concentration is $<0.3 \mathrm{mmol} / \mathrm{L}$, ranging during nutritional ketosis from 0.5 to $3 \mathrm{mmol} / \mathrm{L}$. During prolonged fasting (several weeks), the beta-hydroxybutyrate can increase up to $5-7 \mathrm{mmol} / \mathrm{L}$ (14). Diabetic ketoacidosis is associated with an absolute lack of insulin and includes blood glucose levels usually $>250 \mathrm{mg} / \mathrm{dL}(13.9 \mathrm{mmol} / \mathrm{L})$ accompanied with $\mathrm{pH}$ levels dropping below 7.3 and/or bicarbonate levels $<18 \mathrm{mmol} / \mathrm{L}$ (15).It was shown that the rate of hypoglycemia during Ramadan fasting was $23.8-29.3 \%$ depending on the type of insulin therapy (16); however, most concerning, the rate of severe hypoglycemia was $7.1 \%$ for those using multiple daily insulin injections (MDI). While hypoglycemia is a major obstacle of fasting in people with type 1 diabetes, rates of ketosis were as low as $2.5 \%$ for people on MDI during fasting, assessed in a meta-analysis including 1,699 people with type 1 diabetes (16). Moreover, assuming a change in insulin sensitivity and a potential degradation of hepatic glycogen stores during the fasting period, the question arises if bolus insulin can be applied with a regular carbohydrate-to-bolus insulin ratio at the first carbohydrate intake following the fasting period.

Therefore, the aim of this study was to assess the safety and glycemic parameters during and after prolonged fasting in adults with type 1 diabetes on the short term as a basis for potential future longer studies of intermittent fasting regimens.

\section{METHODS}

This was a single center, cross-over controlled clinical trial assessing the impact of prolonged fasting in adults with type 1 diabetes. The local ethics committee of the Medical University of Graz (Austria) approved the study protocol (30-238 ex 17/18), which was registered at the German Clinical Trials Register (DRKS00016148; DRKS.de). The study was conducted in conformity with the declaration of Helsinki and Good Clinical Practice. Before any trial related activities, potential participants were informed about the study protocol and participants gave their written informed consent.

\section{Eligibility Criteria}

Eligibility criteria included a diagnosis of type 1 diabetes for longer than 12 months, age $\geq 18$ years, treatment with exogenous insulin by means of MDI or continuous subcutaneous insulin infusion (CSII), c-peptide level $\leq 0.3 \mathrm{nmol} / \mathrm{l}$, glycated hemoglobin $\left(\mathrm{HbA}_{1 \mathrm{c}}\right)<80 \mathrm{mmol} / \mathrm{mol}(<9.5 \%)$ and a body mass index (BMI) $20-29.9 \mathrm{~kg} / \mathrm{m}^{2}$. Individuals were not included if they experienced diabetic ketoacidosis or had a history of severe hypoglycemia requiring external assistance within the last 12 months, had a history of any life-threatening disease or any other condition that could influence the study procedures. Additionally, participants had to wear an intermittently scanned continuous glucose monitoring system (isCGM; FreeStyle Libre 1, Abbott, USA) for the assessment of interstitial glucose levels during the fasting period and hypoglycemia was verified by capillary blood glucose measurement.

\section{Assessment of Eligibility}

Inclusion and exclusion criteria were assessed by a study physician at the screening visit, performed at least three days prior to the first fasting period. A venous blood sample was drawn for the assessment of $\mathrm{HbA}_{1 \mathrm{c}}$ and c-peptide levels and general health status was assessed by means of physical examination and determination of complete blood count and urine sample. Cardiovascular status was investigated via resting electrocardiogram and blood pressure measurements.

\section{Fasting Period}

After inclusion in the study, participants were explained to record physical activity and episodes of hypoglycemia $(<70 \mathrm{mg} / \mathrm{dL}$; $<3.9 \mathrm{mmol} / \mathrm{L}$ ) and hyperglycemia $(>270 \mathrm{mg} / \mathrm{dL} ; 15.0 \mathrm{mmol} / \mathrm{L})$ requiring treatment during the fasting period in a diary. Participants using MDI injected their regular basal insulin dose, as discussed in their regular Diabetes Outpatient Clinic visit. Those that were using CSII applied their regular basal insulin 
rate, however, if required, the basal rate was lowered by up to $25 \%$ if deemed necessary by the participants. The fasting period was defined as absolute avoidance of any caloric intake including caffeinated drinks and alcohol, although the consumption of tap water and soda was allowed ad libitum. The amount of consumed water was also recorded in a diary by the participants. The (first) short fasting period lasted 12 hours from 8 PM until 8 AM the next morning. The (second) prolonged fasting period had a duration of 36 hours starting at $8 \mathrm{PM}$. Both fasting periods were separated by at least one week and for both fasting periods, the participants were advised to apply the same amount of basal insulin either in the evening before, in the morning or both.

\section{Trial Visits}

After each fasting period, participants attended the Clinical Research Facility for the assessment of anthropometric parameters, resting metabolic rate (Metamax 3B, Cortex, GER) and a bioelectrical impedance analysis (BIA; BIACORPUS RX 4004M, Medical Health Care GmbH, GER). For the resting metabolic rate, a face mask held in place by a nylon harness covered the participant's nose and mouth. The mask was attached to a bidirectional digital turbine flow meter to measure the volume of inspired and expired air. A sample line between the turbine and analyzer unit determined $\mathrm{O}_{2}$ and $\mathrm{CO}_{2}$ content. A two point calibration procedure was conducted before each testing session according to the manufacturer's guidelines (Calibration Manual 931-00-264/Revision a/2014-03-06, CORTEX Biophysik GmbH, Leipzig, Germany). Participants rested for a period of 20 minutes in the supine position before resting metabolic rate testing, during which breath by breath measurements were recorded for 30 minutes and stability of data were automatically selected by the system.

Afterwards, venous blood samples were drawn for the assessment of fasting glucose, insulin, cortisol, glucagon, betahydroxybutyrate and further metabolic markers. In addition, glucose, insulin, c-peptide, glucagon and beta-hydroxybutyrate levels were assessed over the course of the oral glucose tolerance test (OGTT): immediately before (T1), $15 \mathrm{~min}$ (T2), $30 \mathrm{~min}$ (T3), $60 \mathrm{~min}$ (T4), $120 \mathrm{~min}$ (T5), $180 \mathrm{~min}$ (T6) and $240 \mathrm{~min}$ (T7) after drinking the standardized $75 \mathrm{~g}$ carbohydrate drink (Glucoral, Germania Pharmazeutika, AUT). Immediately before both OGTTs, the same bolus insulin dose based on the regular carbohydrate-to-insulin ratio was injected. Capillary blood glucose measurements were performed for safety reasons in case of fast decreasing or increasing sensor glucose levels. OGTTs were discontinued early if the capillary blood glucose concentration dropped below $70 \mathrm{mg} / \mathrm{dL}(3.9 \mathrm{mmol} / \mathrm{L})$ and immediately $15-30$ gr carbohydrates were orally ingested by the participants.

\section{Outcome Measurements}

We defined the change in 2 hours postprandial glucose during the OGTTs as primary outcome in this study. Additional outcomes included hypoglycemic events, glycemic patterns as assessed by isCGM and during the OGTT as well as body composition.

\section{Statistical Assessments}

All data were assessed for normal distribution by means of Shapiro-Wilk normality testing. Interstitial glucose levels during the fasting period were stratified for time below range level $2(<54 \mathrm{mg} / \mathrm{dL} ;<3.0 \mathrm{mmol} / \mathrm{l})$, time below range level 1 (54-70 mg/dL; 3.0-3.9 mmol/L), time in range $(70-180 \mathrm{mg} / \mathrm{dL}$; $3.9-10.0 \mathrm{mmol} / \mathrm{L})$, time above range level $1(181-250 \mathrm{mg} / \mathrm{dL}$; $10.1-13.9 \mathrm{mmol} / \mathrm{L})$ and time above range level $2(>250$ $\mathrm{mg} / \mathrm{dL}$; $>13.9 \mathrm{mmol} / \mathrm{L})$. Singular post-fasting measurements and area under the curve (AUC; trapezoidal rule) in comparison of overnight vs. prolonged fasting were assessed by means of paired t-test or Mann-Whitney $U$ test. Variables that were investigated over the course of the OGTT were compared between overnight vs. prolonged fasting via two-way ANOVA or mixed-model regressions ( $\mathrm{p} \leq 0.05)$. For the sample size estimation, we assumed a difference in the $2 \mathrm{~h}$-glucose during the OGTT of $20 \pm 25 \mathrm{mg} / \mathrm{dL}(1.1 \pm 1.4 \mathrm{mmol} / \mathrm{L})$ between $12 \mathrm{hrs}$ and $36 \mathrm{hrs}$ fasting. Based on a paired t-test (two-sided, alpha 5\%, power 90\%), 19 participants were required to demonstrate the assumed difference. To compensate potential dropouts, 20 participants were included (Figure 1).

\section{RESULTS}

In total 20 individuals with type 1 diabetes ( 7 females) were included in the study with a mean \pm SD age of $35 \pm 11$ years, BMI $24.8 \pm 2.8 \mathrm{~kg} / \mathrm{m}^{2}, \mathrm{HbA}_{1 \mathrm{c}} 54 \pm 7 \mathrm{mmol} / \mathrm{mol}(7.1 \pm 0.6 \%)$, diabetes duration $20 \pm 11$ years, total daily insulin dose $40 \pm 14 \mathrm{IU}$. Eleven participants were using MDI therapy (6 insulin Aspart (Novo Nordisk, DEN), 4 insulin Lispro (Eli Lilly, USA), 1 faster insulin Aspart (Novo Nordisk, DEN); 5 insulin Degludec (Novo Nordisk, DEN), 6 insulin Detemir (Novo Nordisk, DEN) and 9 were using CSII therapy (2 insulin Lispro (Eli Lilly, USA), 7 insulin Aspart (Novo Nordisk, DEN).

\section{Glycemia During Fasting}

Mean interstitial glucose levels were similar in comparison of the two fasting periods ( $12 \mathrm{hrs}: 138 \pm 35 \mathrm{mg} / \mathrm{dL}[7.7 \pm 1.9 \mathrm{mmol} / \mathrm{L}] v s$. 36 hrs: $130 \pm 17 \mathrm{mg} / \mathrm{dl}[7.2 \pm 0.9 \mathrm{mmol} / \mathrm{L}], \mathrm{p}=0.44)$. Coefficient of variation was numerically but not statistically lower for the short fasting period ( 12 hrs: $25 \pm 10 \%$ vs.. 36 hrs: $31 \pm 9 \%$, p = 0.07). SD of interstitial glucose was not significantly different between both fasting periods $(12 \mathrm{hrs}: 27 \pm 12 \mathrm{mg} / \mathrm{dl}[1.5 \pm 0.7 \mathrm{mmol} / \mathrm{L}]$ vs. 36 hrs: $29 \pm 10 \mathrm{mg} / \mathrm{dL}[1.7 \pm 0.5 \mathrm{mmol} / \mathrm{L}], \mathrm{p}=0.41)$. When data were separated for day- (06:00 AM-12:00 PM) and night time (12:01 PM05:59 AM), a lower mean interstitial glucose concentration was seen during the night time period for the prolonged fasting period (12 hrs: $139 \pm 46 \mathrm{mg} / \mathrm{dL}[7.7 \pm 2.6 \mathrm{mmol} / \mathrm{L}]$ vs. 36 hrs: $112 \pm 22 \mathrm{mg} / \mathrm{dL}$ $[6.2 \pm 1.2 \mathrm{mmol} / \mathrm{L}], \mathrm{p}=0.05)$; furthermore, lower glycemic variability was observed during the prolonged fasting period (12 hrs: $27 \pm 10 v s .36$ hrs: $16 \pm 12 \%, \mathrm{p}=0.01)$. Other interstitial glucose parameters as given in Table 1 remained similar between both trial arms $(\mathrm{p}>0.05)$.

The rate of hypoglycemia per hour over the fasting period defined as levels below $70 \mathrm{mg} / \mathrm{dL}(3.9 \mathrm{mmol} / \mathrm{L})$ was similar in 


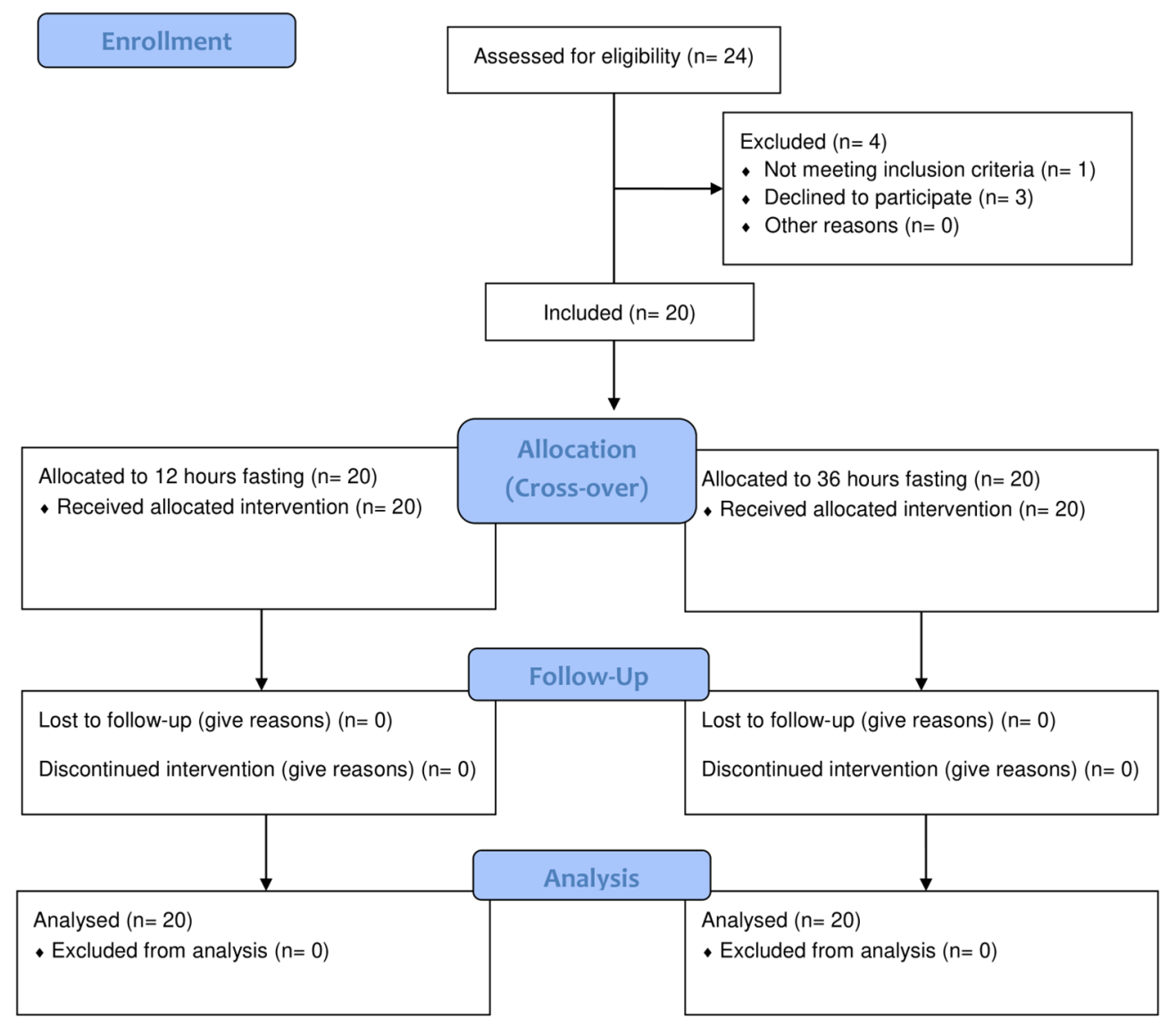

FIGURE 1 | Study flow diagram.

comparison of both trial arms (12 hrs: $0.07 \pm 0.06$ vs. 36 hrs: $0.05 \pm 0.03, \mathrm{p}=0.21)$ facing median and interquartile range interstitial glucose nadir of $60 \mathrm{mg} / \mathrm{dL}$ [ $48-68 \mathrm{mg} / \mathrm{dL}](3.3 \mathrm{mmol} / \mathrm{L}$ [2.7-3.8 mmol/L] for the overnight fasting period and $63 \mathrm{mg} / \mathrm{dl}$ [58-68 mg/dL] $(3.5 \mathrm{mmol} / \mathrm{L}[3.2-3.8 \mathrm{mmol} / \mathrm{L}]$ for the prolonged fasting period $(\mathrm{p}=0.35) .59 \%$ of episodes of hypoglycemia required supplemental carbohydrates during the $12 \mathrm{hrs}$ fasting period (18 gram [15-24]) vs. 71\% during the $36 \mathrm{hrs}$ fasting period (25 gram [15-27] $(\mathrm{p}=0.55)$. Remaining episodes of hypoglycemia, mainly occurring during the night time period and were endogenously regulated. Potentially prandial bolus insulin associated hypoglycemia ( +3 hrs of last dosing) accounted for $17 \%$ of all hypoglycemic episodes in both groups. Those with a higher dose of basal insulin/basal insulin rate had a significantly higher absolute number of hypoglycemic episodes when compared to those with a lower dose during the $36 \mathrm{hrs}$ fasting period (total daily basal insulin dose (TDBD) $<0.25 \mathrm{IU} / \mathrm{kg}$ : $1.33 \pm 0.86 v s$. TDBD $>0.25 \mathrm{IU} / \mathrm{kg}: 2.5 \pm 0.85, \mathrm{p}=0.008)$, but not during the $12 \mathrm{hrs}$ fasting period (TDBD <25 IU/kg: $1.0 \pm 1.8 \mathrm{vs}$. TDBD $>25$ IU/kg: $1.3 \pm 0.95, \mathrm{p}=0.53)$. For both trial arms, the basal insulin rate per hour was similar for CSII (12 hrs fasting: $0.92 \pm 0.18 \mathrm{IU} / \mathrm{hr} v s .36 \mathrm{hrs}$ fasting: $0.88 \pm 0.24 \mathrm{IU} / \mathrm{hr}, \mathrm{p}=0.33$ )

TABLE 1 | Comparison of glycemic ranges for 12 hrs vs. 36 hrs fasting ( $n=20)$.

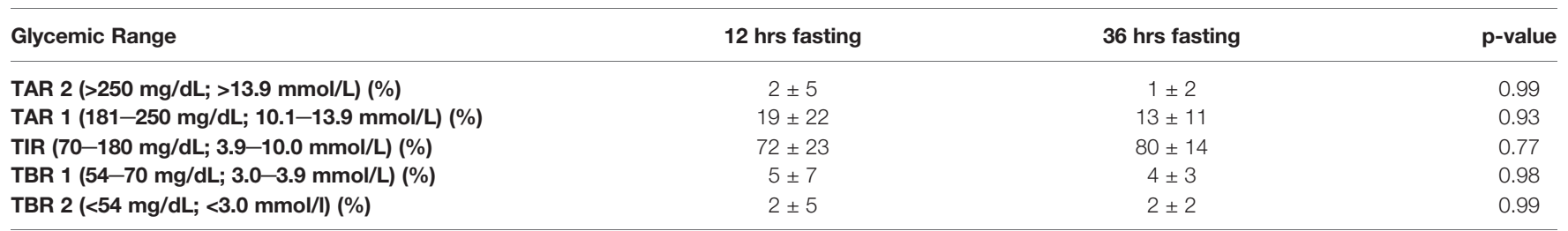

TAR 2, time above range 2; TAR 1, time above range 1; TIR, time in range; TBR 1, time below range 1; TBR 2, time below range $2(n=20)$. 
and identical for MDI (for both trial arms: $0.82 \pm 0.22$ ). No bolus insulin dose was applied in any of the participants during the fasting periods. For both trial arms, all participants were following the fasting regimen, except when carbohydrates were given to treat hypoglycemia. Time spent in the pre-specified glycemic ranges is given in Table $\mathbf{1}$.

\section{Effects of Prolonged Fasting on Resting Metabolic Rate and Anthropometry}

The resting metabolic rate was similar in comparison of both trials arms (12 hrs fasting: $2191 \pm 357 \mathrm{kcal} /$ day vs. $2186 \pm 349$, $\mathrm{p}=0.92$ ), while the respiratory exchange ratio was lower for prolonged fasting (12 hrs fasting: $0.87 \pm 0.05$ (/) vs. 36 hrs: $0.82 \pm 0.03(/), \mathrm{p}=0.001)$ accompanied by higher fat oxidation (12 hrs fasting: $90 \pm 40 \mathrm{~g} /$ day $v s .36 \mathrm{hrs}$ fasting: $130 \pm 35 \mathrm{~g} /$ day, $\mathrm{p}<0.001)$ and lower carbohydrates oxidation following prolonged fasting (12 hrs fasting: $305 \pm 98$ g/day vs. $36 \mathrm{hrs}$ fasting: $215 \pm 63, p=0.007)$. Protein metabolism remained unaffected by prolonged fasting ( $12 \mathrm{hrs}$ fasting: $25 \pm 4 \mathrm{~g} /$ day $v$. 36 hrs fasting: $25 \pm 4, \mathrm{p}=0.63)$. Body weight $(76.7 \pm 13.5 \mathrm{~kg} v s$. $75.4 \pm 13.4 \mathrm{~kg}, \mathrm{p}=0.0002)$ and BMI $(24.6 \pm 2.8 \mathrm{vs} .24 .2 \pm 2.8 \mathrm{~kg} /$ $\mathrm{m} 2 ; \mathrm{p}=0.0001)$ following the prolonged fasting period were significant lower when compared against overnight fasting. Fat mass $(\mathrm{p}=0.26)$, free fat mass $(\mathrm{p}=0.79)$, total body water $(\mathrm{p}=0.46)$ and body cell mass $(\mathrm{p}=0.73)$ were similar after both fasting periods.

\section{Effects of Prolonged Fasting on Metabolic Markers}

Significant differences were found in comparison of post-12 hrs fasting versus post-36 hours fasting for uric acid $(\mathrm{p}<0.001)$, bilirubin $(p=0.002)$, triglycerides $(p<0.001)$, VLDL $(p=0.011)$, serum iron $(\mathrm{p}=0.040)$, ferritin $(\mathrm{p}=0.01)$, transferrin saturation $(\mathrm{p}=0.02)$ and leptin $(\mathrm{p}=0.006)$ which can be found in Table 2.

\section{Effects of Prolonged Fasting on Metabolic and Hormonal Markers During OGTT}

OGTTs were discontinued due to hypoglycemia three times in three people in each trial arm. The median [interquartile range] of the
120 min OGTT plasma glucose AUC were similar in comparison of both fasting regimes (12 hrs fasting 32798 [24791-38831] vs. $36 \mathrm{hrs}$ fasting [30146 [26361-33154]; $\mathrm{p}=0.21)$. Mean $120 \mathrm{~min}$ plasma glucose levels were also similar in comparison of both trial arms (12 hrs fasting $308 \pm 91 \mathrm{mg} / \mathrm{dL}[17.1 \pm 5.1 \mathrm{mmol} / \mathrm{L}]$ vs. 36 hrs fasting $313 \pm 71 \mathrm{mg} / \mathrm{dL}[17.4 \pm 3.9 \mathrm{mmol} / \mathrm{L} ; \mathrm{p}=0.73)$. For the OGTTs, exactly the same dose of bolus insulin was applied ( $6 \pm 2 \mathrm{IU})$ in both trial arms. Over the course of the OGTT, plasma glucose levels significantly increased for both trial arms $(\mathrm{p}<0.001)$, following a similar course $(p=0.04)$. The course of plasma glucose, insulin, glucagon, beta-hydroxybutyrate and cortisol in comparison of both trial arms is detailed in Figure 2. Three episodes of hypoglycemia occurred after the overnight fasting during the OGTT (mean glucose nadir $64 \pm 5 \mathrm{mg} / \mathrm{dL}[3.6 \pm 0.3 \mathrm{mmol} / \mathrm{L}]$ ) and three episodes of hypoglycemia occurred after the prolonged fasting during the OGTT (mean glucose nadir $65 \pm 6 \mathrm{mg} / \mathrm{dL}$ [3.6 $\pm 0.3 \mathrm{mmol} / \mathrm{L}]$ ) - those three people had also an episode during the overnight fasting period (comparison of hypoglycemic events between groups, $\mathrm{p}=0.50$ ).

\section{Differences in MDI Versus CSII Therapy}

During the $12 \mathrm{hrs}$ fasting period, participants using MDI therapy spent more TIR when compared to those using CSII therapy (MDI $83 \pm 15 \%$ vs. CSII $62 \pm 25 \%$; $\mathrm{p}=0.006$ ), which was not seen during the 36 hrs fasting period (MDI $78 \pm 17 \%$ vs. CSII $82 \pm$ $11 \% ; \mathrm{p}=0.74)$. Remaining glycemic ranges, risk of hypoglycemia and insulin therapy were not significantly different in comparison of the type of therapy $(p>0.05)$. During the OGTT for both after the $12 \mathrm{hrs}(\mathrm{p}=0.0002)$ and $36 \mathrm{hrs}$ fasting period ( $p<0.0001$ ), the MDI group had higher insulin levels when compared to the CSII group. Remaining markers assessed during the OGGT where not significantly different in comparison of groups $(\mathrm{p}>0.05)$.

\section{DISCUSSION}

This is the first study investigating the glycemic effects and safety of a $36 \mathrm{hrs}$ prolonged fasting period in adults with type 1

TABLE 2 | Effects of prolonged fasting on metabolic markers $(n=20)$.

\begin{tabular}{|c|c|c|c|}
\hline Glycemic Range & 12 hrs fasting & 36 hrs fasting & p-value \\
\hline Uric acid $(\mathrm{mg} / \mathrm{dL})$ & $4.12 \pm 1.02$ & $4.97 \pm 1.10$ & $<0.001$ \\
\hline Bilirubin (mg/dL) & $0.90 \pm 0.76$ & $1.33 \pm 1.17$ & 0.002 \\
\hline Triglycerides (mg/dL) & $64 \pm 18$ & $80 \pm 28$ & $<0.001$ \\
\hline Cholesterol (mg/dL) & $195 \pm 32$ & $201 \pm 34$ & 0.220 \\
\hline HDL (mg/dL) & $73 \pm 18$ & $71 \pm 20$ & 0.357 \\
\hline LDL (mg/dL) & $102 \pm 34$ & $109 \pm 32$ & 0.140 \\
\hline VLDL (mg/dL) & $14 \pm 4$ & $17 \pm 4$ & 0.011 \\
\hline Serum-Iron $(\mu \mathrm{g} / \mathrm{dL})$ & $125 \pm 54$ & $101 \pm 35$ & 0.040 \\
\hline Ferritin (ng/mL) & $115 \pm 67$ & $135 \pm 82$ & 0.010 \\
\hline Transferrin (g/L) & $2.3 \pm 0.6$ & $2.3 \pm 0.5$ & 0.140 \\
\hline Transferrin saturation (\%) & $42 \pm 22$ & $32 \pm 13$ & 0.020 \\
\hline Gastrin (pg/mL) & $23 \pm 11$ & $23 \pm 8$ & 0.97 \\
\hline Leptin (ng/mL) & $3.47 \pm 3.25$ & $2.42 \pm 2.46$ & 0.006 \\
\hline
\end{tabular}



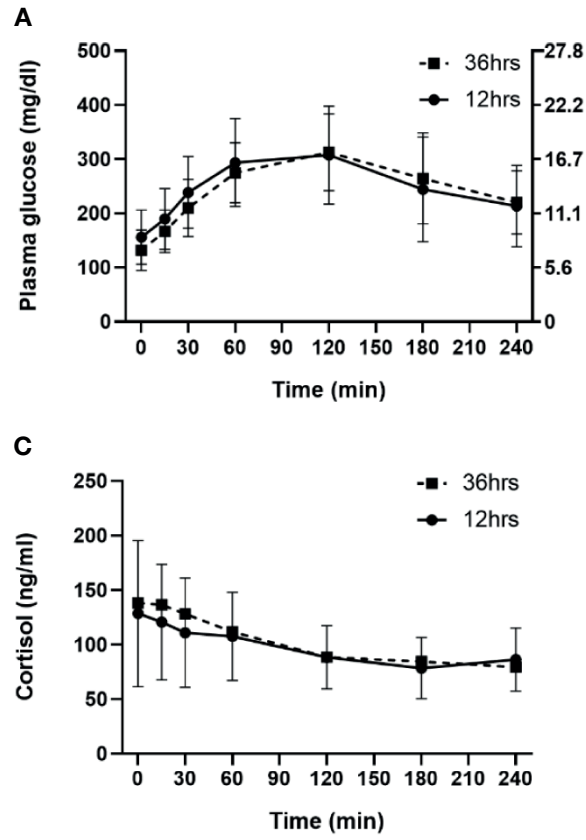

E

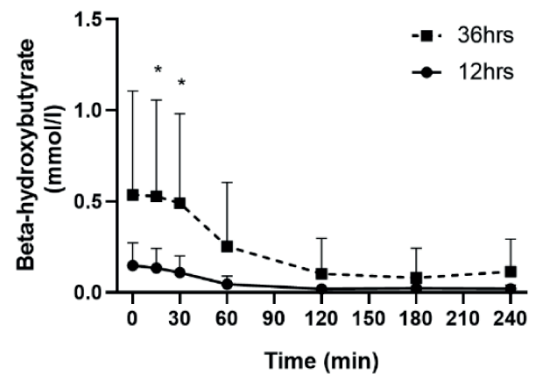

B

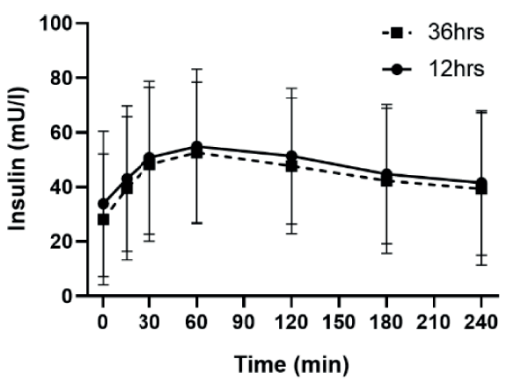

D

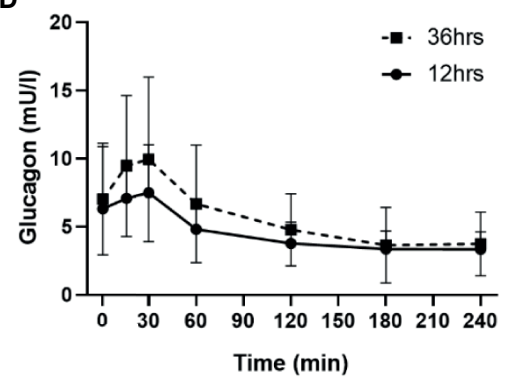

FIGURE 2 | Comparison of 12 hrs fasting vs. 36 hrs fasting for plasma glucose (A), insulin (B), cortisol (C), glucagon (D) and beta-hydroxybutyrate (E). * indicates significant difference for the specific time point $n$ comparison of both trial arms $(n=20)$.

diabetes. Out of our results it might be concluded that prolonged fasting up to $36 \mathrm{hrs}$ is feasible accompanied by a low risk of dysglycemia in people under good glycemic control. A similar conclusion has be drawn in a recent study when a multimodal fasting intervention was performed over a period of 7 days in adults with type 1 diabetes (13). Furthermore, Berger et al. showed that such a prolonged fasting period can maintain euglycemia while $\beta$-hydroxybutyrate levels were elevated by up to $2.8 \mathrm{mmol} / \mathrm{L}$ and bodyweight as well as BMI decreased and were maintained for up to 4 months after the intervention (13).

As concluded in a previous review, insulin resistance to exogenous insulin is deteriorating over the course of type 1 diabetes, which was found to be associated to some extent to bodyweight (17). In the transition from the Diabetes Control and Complications Trial to the Epidemiology of Diabetes Interventions and Complications study, it was shown that excess weight gain was associated with insulin resistance (18). Taking into account that in people with type 1 diabetes exogenous insulin is continuously circulating that is inversely related to rates of lipolysis, safe and feasible strategies need to be defined for bodyweight and insulin resistance management. Even a healthy lifestyle consisting of physical activity and exercise (19, 20) as well as a balanced diet (21), might not solely be sufficient for losing body weight in type 1 diabetes and therefore people are using alternative strategies with significant caloric restriction, requiring withholding bolus insulin doses. Therefore, a solid research basis is required for future patient counselling.

Intriguingly, in our study during the prolonged fasting, the basal insulin rate for CSII was only lowered by $\sim 4 \%$ that was sufficient to prevent hypoglycemia. This finding differs to recent recommendations, in which it was detailed that people with type 1 diabetes should reduce the basal insulin rate initially by $10 \%$ to an extent of as much as $90 \%$ towards the end of the fasting day (22). However, given the nature of our well-controlled and safe study design and the single fasting episode only, a direct comparison to these recommendations might be difficult. Therefore, it is important to point out, that if basal insulin rate is reduced to a greater extent, not only blood glucose should be 
monitored but also ketone levels (23). In line with previous research (24), fasting induced a significant increase in serum uric acid levels that might be evoked by a decrease in uric acid excretion by the kidney in the presence of elevated ketones which were significantly higher (beta-hydroxybutyrate of $0.54 \mathrm{mmol} / \mathrm{l}$ ) after the prolonged fasting period than after 12 hours $(0.15 \mathrm{mmol} /)$. Total serum bilirubin concentration did rise significantly, as it was observed previously (25), however, the mean value was just above the normal range. While gastrin, a hormone that increases the release of gastric acid, remained unchanged by prolonged fasting, leptin, a hormone that regulates the sensation of hunger, significantly decreased, which is in line with a previous study performed in healthy individuals (26).

For both, during the fasting period as well as with the first dosing after the fasting, prolonged fasting can be safely performed in people with well-controlled type 1 diabetes. Numerically, less episodes of hypoglycemia were observed during the prolonged fasting period when compared against the overnight fasting. It can be hypothesized that our participants did more thoroughly monitor their glucose levels before and during the fasting, yielding such a low number of hypoglycemia by consuming carbohydrates. In line with this finding, TIR was not different between overnight fasting and prolonged fasting. From a physiological point of view, one might assume that with the first dosing after a prolonged fasting period the carbohydrates-to-bolus insulin ratio might be lowered due to hepatic glycogen depletion during fasting or potentially via an increased insulin sensitivity. Intriguingly, we did observe the same glycemic excursions during the OGTT in both groups and three episodes of hypoglycemia occurred during the OGTT following prolonged and 12 hours fasting. This finding suggests that people with type 1 diabetes do not need to adapt their bolus insulin dose with the first meal after a prolonged fasting period. In line with the low risk of hypoglycemia in comparison of both trial arms, hormones and metabolic markers showed similar patterns during the OGTT, except beta-hydroxybutyrate. However, these physiologically elevated ketone levels reflect the shift to lipolysis during fasting (27).

Our study is not without limitations: since this was the first study assessing the safety and glycemic effects of prolonged fasting, our study participants were not randomized to the sequence of the 12 hrs and $36 \mathrm{hrs}$, therefore $12 \mathrm{hrs}$ fasting periods were always followed by the 36 hours period. In addition, in case of a hypoglycemic event, participants administered carbohydrates and strictly speaking the fasting period ended at this time point. However, this administration was critical for safety reasons. Furthermore, only participants running on CSII were allowed to lower the basal insulin rate but not those with MDI. Notwithstanding, within the group of participants on CSII the basal insulin rate was similar for both trial arms. While this is important data on prolonged fasting in people with type 1 diabetes, we appreciate that from a single 36 fasting period one cannot extrapolate to the safety of intermittent fasting over a longer period of time in this population, an aspect that clearly needs further attention and investigation. Furthermore, the sample size for assessing the safety and efficacy was in general small but can serve as the basis for sample size estimations in future, larger fasting studies. Additionally, we did not assess the status of the menstrual cycle that might also have an impact on the results. In addition, it clearly needs to be pointed out that an OGTT does not reflect normal nutrition habits after a fasting period; so our procedure was artificially mimicking the first carbohydrate intake after prolonged fasting. For future studies, it is also urgently needed to assess blood ketone levels not only at the end of the fasting period but also continuously during the fasting period.

\section{CONCLUSIONS}

Our proof-of-concept study showed that people with type 1 diabetes under good glycemic control might be able to safely perform a prolonged fasting period with a limited risk of severe dysglycemia. However, we performed a study with a single fasting episode only, which cannot be extrapolated to intermittent fasting interventions of longer duration. Hence, future studies over a more extended period of time are required to detail the entire spectrum of potentially beneficial effects of interventional fasting in people with type 1 diabetes.

\section{DATA AVAILABILITY STATEMENT}

The raw data supporting the conclusions of this article will be made available by the authors, without undue reservation upon request.

\section{ETHICS STATEMENT}

The studies involving human participants were reviewed and approved by the local ethics committee of the Medical University of Graz (Austria) (30-238 ex 17/18). The patients/participants provided their written informed consent to participate in this study.

\section{AUTHOR CONTRIBUTIONS}

OM, ME, NT, PP, and HS designed and performed the study, interpreted data, and contributed to discussions. OM, HY, NG, and FAbe drafted the manuscript and/or performed statistical analysis. OM, ME, AM, NT, HY, FAbe, FAbb, PP, FA, AO, TP, $\mathrm{HK}, \mathrm{CS}, \mathrm{MB}, \mathrm{TN}, \mathrm{MH}$, and HS performed the study. All authors contributed to the article and approved the submitted version.

\section{FUNDING}

This study was supported by the Austrian Science Fund (KLIF-851).

\section{ACKNOWLEDGMENTS}

We want to thank the participants for their significant adherence to the study protocol. 


\section{REFERENCES}

1. de Cabo R, Mattson MP. Effects of Intermittent Fasting on Health, Aging, and Disease. N Engl J Med (2019) 381:2541-51. doi: 10.1056/nejmra1905136

2. Mattson MP, Longo VD, Harvie M. Impact of Intermittent Fasting on Health and Disease Processes. Ageing Res Rev (2017) 39:46-58. doi: 10.1016/ j.arr.2016.10.005

3. Varady KA, Hellerstein MK. Alternate-Day Fasting and Chronic Disease Prevention: A Review of Human and Animal Trials. Am J Clin Nutr (2007) 86:7-13. doi: 10.1093/ajcn/86.1.7

4. Rynders CA, Thomas EA, Zaman A, Pan Z, Catenacci VA, Melanson EL. Effectiveness of Intermittent Fasting and Time-Restricted Feeding Compared to Continuous Energy Restriction for Weight Loss. Nutrients (2019) 11 (10):2442 (1-23). doi: 10.3390/nu11102442

5. Stekovic S, Hofer SJ, Tripolt N, Aon MA, Royer P, Pein L, et al. Alternate Day Fasting Improves Physiological and Molecular Markers of Aging in Healthy, Non-Obese Humans. Cell Metab (2019) 30:462-76. doi: 10.1016/j.cmet.2019.07.016

6. Di Francesco A, Di Germanio C, Bernier M, De Cabo R. A Time to Fast. Sci (80-) (2018) 362:770-5. doi: 10.1126/science.aau2095

7. Furmli S, Elmasry R, Ramos M, Fung J. Therapeutic Use of Intermittent Fasting for People With Type 2 Diabetes as an Alternative to Insulin. BMJ Case Rep (2018) 2018:1-5. doi: 10.1136/bcr-2017-221854

8. Sutton EF, Beyl R, Early KS, Cefalu WT, Ravussin E, Peterson CM. Early Time-Restricted Feeding Improves Insulin Sensitivity, Blood Pressure, and Oxidative Stress Even Without Weight Loss in Men With Prediabetes. Cell Metab (2018) 27:1212-1221.e3. doi: 10.1016/j.cmet.2018.04.010

9. Mattson MP, Moehl K, Ghena N, Schmaedick M, Cheng A. Intermittent Metabolic Switching, Neuroplasticity and Brain Health. Nat Rev Neurosci (2018) 19:81-94. doi: 10.1038/nrn.2017.156

10. Deeb A, Al Qahtani N, Akle M, Singh H, Assadi R, Attia S, et al. Attitude, Complications, Ability of Fasting and Glycemic Control in Fasting Ramadan by Children and Adolescents With Type 1 Diabetes Mellitus. Diabetes Res Clin Pract (2017) 126:10-5. doi: 10.1016/j.diabres.2017.01.015

11. Fellinger P, Fuchs D, Wolf P, Heinze G, Luger A, Krebs M, et al. Overweight and Obesity in Type 1 Diabetes Equal Those of the General Population. Wien Klin Wochenschr (2019) 131:55-60. doi: 10.1007/s00508-018-1434-9

12. Reiter J, Wexler ID, Shehadeh N, Tzur A, Zangen D. Type 1 Diabetes and Prolonged Fasting. Diabetes Med (2007) 24:436-9. doi: 10.1111/j.14645491.2007.02098.x

13. Berger B, Jenetzky E, Köblös D, Stange R, Baumann A, Simstich J, et al. SevenDay Fasting as a Multimodal Complex Intervention for Adults With Type 1 Diabetes: Feasibility, Benefit and Safety in a Controlled Pilot Study. Nutrition (2021) 86:1-16. doi: 10.1016/j.nut.2021.111169

14. Owen OE. Ketone Bodies as a Fuel for the Brain During Starvation. Biochem Mol Biol Educ (2005) 33:246-51. doi: 10.1002/bmb.2005.49403304246

15. Kitabchi AE, Umpierrez GE, Miles JM, Fisher JN. "Hyperglycemic Crises in Adult Patients With Diabetes". In: Diabetes Care. American Diabetes Association Diabetes Care (2009) 32(7)1335-43. doi: 10.2337/dc09-9032

16. Loh HH, Lim LL, Loh HS, Yee A. Safety of Ramadan Fasting in Young Patients With Type 1 Diabetes: A Systematic Review and Meta-Analysis. J Diabetes Investig (2019) 10:1490-501. doi: 10.1111/jdi.13054

17. Bjornstad P. Insulin Sensitivity and Complications in Type 1 Diabetes: New Insights. World J Diabetes (2015) 6:8. doi: 10.4239/wjd.v6.i1.8

18. Purnell JQ, John EH, Cleary PA, Nathan DM, Lachin JM, Zinman B, et al. The Effect of Excess Weight Gain With Intensive Diabetes Mellitus Treatment on
Cardiovascular Disease Risk Factors and Atherosclerosis in Type 1 Diabetes Mellitus: Results From the Diabetes Control and Complications Trial/ Epidemiology of Diabetes Interventions and Complications Study (DCCT/ EDIC) Study. Circulation (2013) 127:180-7. doi: 10.1161/CIRCULATIONAHA 111.077487

19. Moser O, Riddell MC, Eckstein ML, Adolfsson P, Rabasa-Lhoret R, van den Boom L, et al. Glucose Management for Exercise Using Continuous Glucose Monitoring (CGM) and Intermittently Scanned CGM (isCGM) Systems in Type 1 Diabetes: Position Statement of the European Association for the Study of Diabetes (EASD) and of the International Society for Pediatric and Adolescent Diabetes (ISPAD) Endorsed by JDRF and Supported by the American Diabetes Association (ADA). Pediatr Diabetes (2020) 21:1375-93. doi: $10.1111 /$ pedi.13105

20. Moser O, Riddell MC, Eckstein ML, Adolfsson P, Rabasa-Lhoret R, van den Boom L, et al. Glucose Management for Exercise Using Continuous Glucose Monitoring (CGM) and Intermittently Scanned CGM (isCGM) Systems in Type 1 Diabetes: Position Statement of the European Association for the Study of Diabetes (EASD) and of the International Society for Pediatric and Adolescent Diabetes (ISPAD) Endorsed by JDRF and Supported by the American Diabetes Association (ADA). Diabetologia (2020) 63:2501-20. doi: 10.1007/s00125-020-05263-9

21. Chiang JL, Kirkman MS, Laffel LMB, Peters AL. Type 1 Diabetes Through the Life Span: A Position Statement of the American Diabetes Association. Diabetes Care (2014) 37:2034-54. doi: 10.2337/dc14-1140

22. Grajower MM, Horne BD. Clinical Management of Intermittent Fasting in Patients With Diabetes Mellitus. Nutrients (2019) 11:1-11. doi: 10.3390/ nu1 1040873

23. Lloyd-Mostyn RH, Lord PS, Glover R, West C, Gilliland IC. Uric Acid Metabolism in Starvation. Ann Rheum Dis (1970) 29:553-5. doi: 10.1136/ $\operatorname{ard}$.29.5.553

24. Ogryzlo MA. Hyperuricemia Induced by High Fat Diets and Starvation. Arthritis Rheum (1965) 8:799-822. doi: 10.1002/art.1780080443

25. Meyer B, Scholtz H, Schall R, Muller F, Hundt H, Maree J. The Effect of Fasting on Total Serum Bilirubin Concentrations. Br J Clin Pharmacol (1995) 39:169-71. doi: 10.1111/j.1365-2125.1995.tb04424.x

26. Chan JL, Heist K, DePaoli AM, Veldhuis JD, Mantzoros CS. The Role of Falling Leptin Levels in the Neuroendocrine and Metabolic Adaptation to Short-Term Starvation in Healthy Men. J Clin Invest (2003) 111:1409-21. doi: $10.1172 /$ jci17490

27. Encyclopedia of Human Nutrition . Available at: https://www.elsevier.com/ books/encyclopedia-of-human-nutrition/unknown/978-0-12-375083-9 (Accessed May 26, 2021).

Conflict of Interest: The authors declare that the research was conducted in the absence of any commercial or financial relationships that could be construed as a potential conflict of interest.

Copyright (c) 2021 Moser, Eckstein, Mueller, Tripolt, Yildirim, Abbas, Pferschy, Goswami, Aberer, Obermayer, Pieber, Kojzar, Souri, Brunner, Niedrist, Herrmann and Sourij. This is an open-access article distributed under the terms of the Creative Commons Attribution License (CC BY). The use, distribution or reproduction in other forums is permitted, provided the original author(s) and the copyright owner(s) are credited and that the original publication in this journal is cited, in accordance with accepted academic practice. No use, distribution or reproduction is permitted which does not comply with these terms. 